\title{
Parole d'étudiants
}

\section{Les limitations et arrêts de traitement en réanimation}

\author{
Withholding and withdrawing life-sustaining treatment in intensive care
}

\author{
Marie SONNIER ${ }^{1}$, Bodo RAKOTOSEHNO ${ }^{1}$, Catherine MAHE ${ }^{1}$, Corry DROGUET ${ }^{1}$ \\ Anne RENAULT'2 Jean-Michel BOLES ${ }^{2}$
}

\begin{abstract}
Résumé Contexte : En France, les dispositions réglementaires prévoient que les étudiants hospitaliers préparent un exposé oral annuel consacré à l'analyse d'une problématique de santé rencontrée au cours de leurs stages. Exégèse : La présente contribution rapporte dans un premier temps le récit narratif et réflexif d'une partie de l'expérience de stage en réanimation de quatre étudiantes, tel quelles l'ont élaboré à l'occasion de leur exposé de fin de stage. Ce récit est ensuite commenté par deux des enseignants cliniciens impliqués dans leur encadrement pendant le stage. Conclusion : L'expérience rapportée illustre l'intérêt des étudiants pour la réflexion éthique concernant les pratiques médicales, dès lors que le contexte de stage est explicitement exploité pour orienter et soutenir des apprentissages intégrant de multiples champs de savoirs.
\end{abstract}

Mots clés Ethnique ; externat ; réanimation ; récit.

Abstract Background: French regulations foresee that medical students perform an annual oral presentation devoted to the analysis of a health problem encountered during their clerkship. Exegesis: This contribution first reports a narrative reflection about part of the clerkship experience in intensive care of four students, as elaborated for their oral presentation. Subsequently, two teaching clinicians involved in the clinical supervision of these students comment their writings. Conclusion: This experience illustrates students' interest in ethical debate about medical practices, so long as clerkship context is explicitly used to guide and support learning integrating multiple fields of knowledge.

Key words Ethnic; clerkship; intensive care; narrative.

Pédagogie Médicale 2008;9:54-61

\footnotetext{
1- Etudiantes (quatrième année du deuxième cycle des études médicales) - Faculté de médecine et des sciences de la santé Université de Bretagne Occidentale, Brest (France).

2- Service de Réanimation médicale et Urgences Médicales - Hôpital de la Cavale Blanche - Centre hospitalier universitaire (CHU) et Equipe reconnue par le conseil scientifique (ERCS A02) "Ethique, professionnalisme et santé » - Département de Sciences Humaines et Sociales - Faculté de Médecine et des Sciences de la Santé - Université de Bretagne Occidentale, Brest (France).

Correspondance : Jean-Michel Boles - Service de Réanimation médicale et Urgences Médicales - Hôpital de la Cavale Blanche -

CHU Boulevard Tanguy Prigent - 29609 Brest Cedex (France) - Téléphone : + 33 (0)2 98347181.

Mailto:jean-michel.boles@chu-brest.fr
} 


\section{Introduction}

En France, les dispositions réglementaires qui encadrent les activités de stage d'externat prévoient qu' " au cours de ces trois années, les étudiants doivent préparer au moins trois exposés sur les sujets en rapport avec la pathologie rencontrée dans les services où ils sont affectés. Ces exposés sont préparés par petits groupes, sous la direction d'un enseignant, à partir de documents et de références bibliographiques réunis à leur intention. Un de ces exposés au moins doit avoir un rapport avec l'évaluation des pratiques de soins, ou avec des problèmes de santé rencontrés aux urgences ou dans d'autres structures dans lesquelles sont dispensés des soins primaires. Ces exposés ont pour objet de développer les capacités d'analyse, de synthèse et de communication orale des étudiants. "'.

A la faculté de médecine de Brest, l'application de ces dispositions répond aux règles suivantes : la présentation de l'exposé oral annuel a lieu pendant le premier stage de chacune des trois années d'externat concernées, qui se déroule du début septembre à la fin novembre ; le stage obligatoire aux urgences, d'une durée de six semaines, est effectué pendant la quatrième et dernière année du deuxième cycle des études médicales (DCEM4) prégraduées; il est couplé avec un stage de six semaines dans l'un des deux services de réanimation, chirurgicale ou médicale. Pendant l'année universitaire 2007-2008, vingt-deux étudiants sont affectés dans le stage urgences - réanimation tous les trimestres et, parmi eux, six passent en réanimation médicale par rotation toutes les six semaines.

Conformément aux modalités en vigueur dans tous les hôpitaux universitaires français, les stages d'externat en réanimation médicale se déroulent exclusivement le matin, l'après-midi étant dévolue aux activités d'enseignement théoriques à la faculté ; cependant, avec l'accord des étudiants, l'un d'entre eux effectue à tour de rôle son stage l'après-midi, enchaînant éventuellement avec la garde le soir. Au cours du stage, treize séances répondant aux principes et au format des séances d'apprentissage du raisonnement clinique ${ }^{2}$ sont organisées. Les étudiants sont libres du choix de leur sujet d'exposé oral annuel, qui doit cependant être agréé par un responsable enseignant du service. Deux à quatre étudiants peuvent choisir le même sujet pour en présenter les différents aspects. Des ressources bibliographiques succinctes leur sont fournies et un accès à internet est par ailleurs disponible dans le service. Les étudiants affectés dans le stage urgences-réanimation présentent leur exposé oral devant l'ensemble des étudiants du stage et les médecins des différentes unités disponibles. Chaque exposé dure 10 à 15 minutes; il est effectué avec le support visuel d'une présentation PowerPoint ${ }^{\circledR}$ et est suivi de remarques rétroactives de la part des enseignants présents et d'une discussion.

Lors du stage de l'automne 2007, quatre étudiantes, âgées de vingt-trois ans, ont fait part de leur souhait de pouvoir choisir un sujet qui ne soit pas strictement en lien avec des aspects biocliniques. Après discussion, le sujet de la problématique des limitations et arrêts de traitements en réanimation a été retenu. Il s'agit en effet d'une problématique fréquente, qui est abordée à de nombreuses occasions durant le stage (visite des malades, réunions de discussion et de synthèse des dossiers, entrevues avec les familles). Trois textes de référence leur ont été fournisis.5.

La présente contribution rapporte dans un premier temps le récit narratif et réflexif d'une partie de l'expérience de stage de ces quatre étudiantes, tel qu'elles l'ont élaboré à l'occasion de leur exposé de fin de stage. Ce récit est ensuite commenté par deux des enseignants cliniciens impliqués dans leur encadrement pendant le stage.

\section{Récit}

\section{Un stage bien différent des autres}

Quinze octobre 2007. "Nous y sommes!». C'est le premier jour de notre stage d'externe en réanimation. Qu'est-ce qui nous attend? Comment allons-nous réagir dans un environnement si inhabituel? Nous " débarquons " littéralement dans un monde à part, inconnu. On nous a prévenus : ce ne sera pas facile...

Dès les premiers jours, nous voici face à une problématique à laquelle nous étions très peu confrontées jusqu'alors mais quasi-quotidienne ici, en réanimation: jusqu'où peut-on aller pour tenter de guérir une personne? Quand atteinton les limites de la démarche médicale? Quand doit-on arrêter une thérapeutique active? Autant de questions peu évidentes pour nous et qui entraînent de multiples interrogations, parfois même une incompréhension : ne nous a ton pas appris que le médecin doit tout mettre en ceuvre pour guérir son patient? Le décès n'est-il pas synonyme d'échec? En arrêtant de traiter quelqu'un, ne l'abandonne-t-on pas? Une longue réflexion s'engage pour chacun d'entre nous. Nos avis s'accordent ou divergent, peu importe, l'important est de dialoguer, partager nos points de vue, pour finalement comprendre que le but de la réanimation est autre chose que maintenir la vie à tout prix. 


\section{Parole d'étudiants}

En réanimation, la prise en charge differe en de nombreux points de celle mise en auvre dans un service de médecine "classique ". En effet, le nombre important de décès conduit à se poser quotidiennement la question de la limitation ou de l'arrêt de thérapeutiques actives. Ceci implique un langage spécifique qu'il nous a fallu maîtriser rapidement : "abstention de traitement ", "arrêt de traitement ", "limitation de traitement ", "soins de confort " En effet, comment prendre part au débat, comprendre les principes et enjeux d'une discussion si on ne sait pas exactement de quoi on parle?

Plusieurs définitions sont admises pour les différents termes. Nos lectures nous ont permis de dégager quelques éléments fondamentaux : lorsqu'on "limite " ou qu'on "arrête " un traitement, il s'agit exclusivement des traitements curatifs ou de suppléance d'organe et en aucun cas des soins de confort (soins d'hygiène, analgésie, hydratation, lutte contre l'angoisse, ...), qui seront délivrés au patient jusqu'à ses derniers instants (devoir légal et moral). Une limitation ou un arrêt des thérapeutiques actives restitue à la mort son caractère naturel: on sait qu'en limitant ou en arrêtant le traitement, le moment de la mort peut être avancé mais que l'intention n'est absolument pas de la provoquer délibérément. Ce point est essentiel à comprendre car c'est ce qui différencie fondamentalement la limitation ou l'arrêt des thérapeutiques actives de l'euthanasie.

Dans quelles circonstances discute t-on une limitation ou un arrêt de thérapentique active? En premier lieu, lorsque le patient le demande puisque c'est à lui qu'appartient la décision de se faire soigner ou non; cette situation est plutôt rare en réanimation du fait de la perte d'autonomie $d u$ patient. La deuxième circonstance possible est l'échec thérapeutique: continuer le traitement ne ferait que retarder le décès de quelques heures ou quelques jours. Enfin, la troisième situation est l'impasse thérapeutique : c'est le cas le plus fréquent en réanimation. Cela signifie que si l'on poursuit les traitements curatifs, on peut sauver le patient de l'issue mortelle mais au prix d'un pronostic ultérieur catastrophique avec une dépendance prévisible très lourde et une qualité de vie plus quiindésirable.

Arrêter un traitement devient parfois la "moins mauvaise " des alternatives pour le patient. La question fondamentale qu'on se pose alors est de savoir si cet acte est éthique ou non. Un certains nombres de critères paraissent indispensables pour affirmer que la limitation ou l'arrêt de thérapeutiques actives est acceptable : le premier de ces critères est le respect des choix du patient. Si le patient ne peut les exprimer lui-même, il faut rechercher activement l'existence de directives anticipées, contacter la personne de confiance si elle existe ou, à défaut, la famille et les proches. Tenter de savoir ce que le patient aurait souhaité dans la situation présente est fondamental. Ensuite, la décision de limitation ou d'arrêt de thérapeutiques actives doit nécessairement être l'aboutissement d'une réflexion menée de manière rationnelle et cohérente et surtout de façon collégiale. L'intention ne doit pas être de donner la mort au patient.

Les cinq principes éthiques fondamentaux doivent être respectés : autonomie du patient (c'est-à-dire est-ce que la démarche thérapeutique respecte le souhait du patient? -loi du 4 Mars 2002), non malfaisance ("primum non nocere", d'abord ne pas nuire), bienfaisance (ce qu'on envisage de faire est-il bénéfique et bon pour la personne?), proportionnalité (la stratégie thérapeutique est-elle proportionnelle à la situation globale du patient ?), justice distributive (la prise en charge du patient est-elle justifiée et n'empêche t-elle pas une autre personne de profiter de soins qui seraient réellement bénéfiques pour elle ?).

D'une façon générale, la démarche médicale bénéficie d'un encadrement légal important. En France, la limitation ou l'arrêt de thérapeutiques actives est l'objet d'un certain nombre de lois inscrites dans le code de la santé publique et le code de déontologie médicale. La loi la plus récente et spécifique est la loi Léonetti du 22 avril 2005. Celle-ci affirme clairement le caractère non acceptable de l'acharnement thérapeutique, l'obligation d'information du patient, l'obligation de respect des choix de ce dernier, son droit d'accès aux soins palliatifs et le devoir du médecin de les lui assurer.

La limitation ou l'arrêt de thérapeutiques actives est donc une démarche éthiquement et légalement acceptable. Mais qui en prend l'initiative? Qui propose d'ouvrir la discussion? Tout membre du personnel soignant ou de la famille du patient peut faire part de son avis sur la démarche thérapeutique du patient et donc engager le processus de réflexion de limitation ou d'arrêt de thérapeutiques actives. En pratique, c'est plus souvent le médecin référent du patient qui propose de se réunir dans le cadre d'une réunion dédiée à cette discussion - dite communément "staff éthique " dans le service - afin de s'accorder sur la meilleure solution (ou la moins mauvaise...) de prise en charge pour son malade.

Nous l'avons compris, la limitation ou l'arrêt de thérapeutiques actives est une réalité indissociable du service de réanimation. Elle répond à des critères éthiques clairement définis et est encadrée légalement de façon précise. En pratique, elle reste une décision difficile à prendre, qui nécessite rigueur, collégialité et sérénité.

\section{Le "staff éthique "}

Le processus décisionnel de limitation ou d'arrêt de thérapeutiques actives passe nécessairement par la tenue d'une 


\section{Les limitations et arrêts de traitement en réanimation}

réunion de stafféthique multidisciplinaire. Celle-ci permet d'aboutir à une décision de prise en charge dans des cas difficiles du point de vue médical, éthique et moral. Tous les membres de l'équipe soignante médicale et paramédicale sont invités à participer à cette réunion, y compris nous, les externes.

Le "stafféthique " s'organise de manière rigoureuse:

- le premier temps consiste à recenser l'ensemble des personnes présentes et à inscrire leurs noms sur le compte rendu de staff.

- Ensuite vient l'exposition du problème de santé de la personne dont la prise en charge pose des difficultés. L'ensemble de l'histoire médicale du patient est détaillé, de même que son état physique et cognitif, son mode de vie avant l'hospitalisation. Sa situation familiale est précisée. Les informations données à la famille sur l'état du patient sont également explicitées.

- Chacune des solutions envisagées pour le patient est confrontée aux cinq principes éthiques fondamentaux qui sont, pour rappel, l'autonomie, la non malfaisance, la bienfaisance, la justice distributive et la proportionnalité.

- La discussion aboutit à une décision claire de limitation ou de poursuite de traitement curatif.

- Une fois la décision prise, sont étudiées ses modalités de mise en cuvre. Par exemple, dans le cas d'un arrêt de traitement: que va-t-on arrêter? Va-t-on l'extuber? Quand? Les soins de confort seront bien entendus poursuivis.

- Enfin, la décision finale (ainsi que la responsabilité) revient au médecin référent du patient.

En faisant des recherches sur Internet, nous avons trouvé qu'à Paris dans certains cas plus difficiles (pour des raisons de culture, de religion par exemple), on peut faire appel au Centre d'éthique clinique. Ce centre, crée en 2002 à l'hôpital Cochin à Paris, propose une aide à la décision dans des situations éthiquement difficiles. Il présente la particularité d'être accessible à tous (patient, famille, équipe soignante). Son déroulement s'effectue en trois temps:

- tout d'abord, des entretiens individuels ont lieu avec les différentes personnes concernées par la décision. Ils sont menés par un binôme soignant - non soignant afin de cerner les enjeux médicaux et non médicaux.

- Ensuite se déroule le "staff» d'éthique clinique : y participent les soignants mais aussi des personnes non soignantes qui peuvent être des juristes, philosophes, sociologues, théologiens, qui sont des représentants de la société civile. Le cas est exposé, les questions éthiques sont soulevées et discutées puis un tour de table fait le point.

- Enfin, la dernière étape se déroule en entretiens individuels avec si possible les mêmes binômes qu'initialement. Ce centre d'éthique clinique est donc multidisciplinaire, ce qui donne une valeur ajoutée à la discussion éthique. Il est important de savoir que ce centre n'a aucune vocation décisionnelle; il donne simplement un avis sur une question posée. Les discussions se rapportent à un cas précis, ne créant ainsi aucun "standard décisionnel " et le médecin référent n'est pas désinvesti de son rôle d'acteur ultime de la décision.

Ces différents "staffs éthiques", quelles que soient leur nature et leur procédure, permettent de recentrer la problématique sur la personne en prenant en compte non seulement l'aspect médical du problème, mais aussi la situation familiale, sociale, culturelle et religieuse. Ils permettent également de prendre la décision dans le calme, en prenant le temps qu'il faut et en mettant à plat les différentes questions soulevées. Enfin, la collégialité permet de "dépassionner " la décision de limitation ou d'arrêt de traitement, parfois difficile à vivre pour les personnes impliquées. Car bien sûr, la prise en charge de réanimation (notamment dans le cadre d'une limitation ou d'arrêt de thérapeutiques actives) est génératrice de souffrance pour le patient et sa famille, mais également pour les soignants.

\section{Nos réactions face à la limitation ou l'arrêt de traitement en médecine :}

Le stage en service de réanimation reste particulier pour nous tous, externes en médecine. Il nous a tous marqués, étonnés, parfois bouleversés. Tout d'abord du fait de l'omniprésence de la mort avec ce nombre important de décès. En effet, la mort reste un sujet peu abordé au cours de nos enseignements théoriques et, quand c'est le cas, on nous apprend avant tout à lutter contre elle et à sauver des vies, la mort n'étant le plus souvent qu'une complication parmi les autres. Ce stage nous rappelle que nous ne sommes pas tout puissants et que la mort est un processus naturel quil faut accepter. Souvent, les patients ont été intubés et sédatés avant notre arrivée. Tout cet appareillage, ainsi que le fait de ne pas pouvoir parler au patient, font quill est difficile de tisser un lien avec lui. Cela créé une certaine distance qui, finalement, même si les patients restent à nos yeux des êtres humains dont la mort n'est pas facile à accepter, nous épargne un peu lorsquils nous quittent.

Le "staff éthique" nous aide également à accepter la mort d'un patient. Nous nous sommes senties bien intégrées à ces réunions, ayant notre avis à donner (consultatif et non décisionnel) et pouvant poser toutes les questions que nous soubaitions. Au bout du compte, la décision prise nous paraissait toujours être la meilleure pour le patient. Nous la comprenions et donc nous l'acceptions, même lorsqu'il s'agissait d'une limitation ou d'un arrêt de traitement.

Les décès que nous avons eu du mal à accepter, tout comme les autres membres de l'équipe soignante d'ailleurs, étaient ceux qui n'étaient pas attendus ou anticipés, comme cette 


\section{Parole d'étudiants}

jeune femme de 32 ans qui est décédée trois jours après son accouchement sans avoir pu voir son bébé.

Une autre chose nous a déstabilisées: il s'agit de l'impossibilité de communiquer avec le patient alors que la base de notre relation avec le patient est la communication! Il y a également cet aspect hypertechnique qui entoure le patient. Lorsque nous entrons dans la chambre d'un patient en début de stage, nous adoptons presque tous la même démarche: nous nous approchons, tout doucement, sur la pointe des pieds, évitant au maximum de faire du bruit, chuchotant plutôt que parlant, tout cela dans le but inconscient de ne pas déranger quelqu'un en plein sommeil. S'ensuit une longue période d'observation: les appareils, les tuyaux, les feuilles de traitement et de surveillance... et, au milieu de tout cela, le patient, si vulnérable et pourtant si intimidant. Les interrogations suivent: à quoi servent tous ces appareils? Est-ce quil souffre? Est-ce quil peut mientendre? Il est relié de toute part à des machines nombreuses et complexes qui se mettent à sonner régulièrement ce qui crée une ambiance très stressante et nous fait perdre nos moyens, sans compter le sentiment de culpabilité quand une alarme se met à sonner par notre faute. Vient le moment où il faut l'examiner: certains externes le font le plus vite possible, sans regarder le patient, sans lui parler. D'autres lui prennent la main et lui expliquent ce qu'ils sont en train de faire. Deux attitudes extrêmes, aucune à blâmer, car nous agissons au mieux, avec notre vécu et nos angoisses.

Toutes ces raisons font naître en nous un sentiment de malaise, d'autant plus qu'on a le sentiment d'être invasif et intrusif en examinant ce patient, qui ne nous voit pas et ne nous entend pas et ne peut donc pas exprimer son consentement, ne serait-ce qu'à notre présence. On a peur de lui faire mal, de le bafouer, de ne pas respecter sa dignité d'être humain. On ressent parfois l'impression désagréable d'une personne "chosifiée ", réduite à un simple corps perfusé, intubé, prélevé et ponctionné, privée de toute intimité et autonomie. On parle même souvent d'elle sans la considérer présente : elle est "là sans être là». Ce sentiment de "déshumanisation " est sans doute exacerbé pour nous, étudiants, qui ne voyons pas les familles et proches.

Enfin, l'age souvent jeune des patients rend leur fin de vie précoce encore moins naturelle et donc moins facilement acceptable. L'émotion est d'autant plus grande que l'on a tendance à sidentifier plus facilement à des personnes qui ont notre âge ou l'age de nos proches.

Le "staff éthique" a également été une découverte pour nous. Il nous a permis de réaliser à quel point la prise en charge d'un patient entre la vie et la mort peut être complexe et à quel point elle nécessite une réflexion bien menée et collective. L'encadrement éthique et légal très structuré permet de faciliter le débat. Le processus décisionnel est grandement aidé par les repères que constituent les cinq principes éthiques fondamentaux que sont la bienfaisance, la non malfaisance, l'autonomie, la proportionnalité et la justice distributive et qui constituent le fil directeur de la réflexion. Le "staff éthique " est un moment de réflexion collective où chacun a le droit d'exprimer son avis, voire son désaccord. Cela pousse toujours plus loin notre réflexion personnelle et nous renvoie à nos propres angoisses vis-à-vis de la mort, de la maladie et de la souffrance.

En réanimation, on parle de limitation ou d'arrêt de traitement : peut-on considérer que ces deux processus reviennent au même? D'un point de vue éthique oui, mais en pratique cela n'est pas si évident. En effet, limiter un traitement revient à s'abstenir d'un geste curatif tandis qu'arrêter un traitement consiste en une action, par exemple l'extubation, qui pour celui qui la pratique engage davantage sa conscience, d'autant plus que la mort peut survenir dans les minutes qui suivent.

Malgré ce contexte difficile, émergent des valeurs fortes auxquelles se raccrocher. Il n'y a pas de tabou de la mort, nous avons pu en parler librement, sans crainte d'être jugées. Chacun des membres de l'équipe a fait preuve d'une grande tolérance face à nos réflexions et à nos réactions et nous avons ressenti un fort esprit de solidarité au sein de l'équipe soignante. De plus, le sens de l'humour de certains permet de détendre l'atmosphère parfois pesante. Par ailleurs, nous avons compris qu'arrêter ce n'est pas échouer! C'est finalement se souvenir et accepter que la mort fait partie de la vie et que la repousser à tout à prix n'est pas toujours synonyme de guérison ou de respect du patient. Nous avons découvert une médecine qui accepte ses limites, qui ne s'entête pas inutilement au détriment du malade. Non, l'arrêt de traitement n'est pas une démission, ni un abandon, c'est un acte difficile qui demande du courage de la part des personnes impliquées.

\section{Commentaires}

Ce récit d'une expérience de stage en milieu de réanimation, élaboré conjointement par quatre étudiantes à l'occasion d'une activité académique formelle de fin de stage, illustre plusieurs aspects pédagogiques intéressants concernant la place des stages cliniques d'externat au sein d'un curriculum de formation médicale initiale. Nous en commenterons quatre : la pertinence et la richesse du stage en tant que contexte de formation pour orienter des apprentissages dédiés au développement des compétences professionnelles; l'importance de rendre explicite le compagnonnage au cours des stages ; la contribution des expériences de stage au pro- 


\section{Les limitations et arrêts de traitement en réanimation}

cessus de socialisation professionnelle; l'intérêt de l'écriture pour soutenir des apprentissages dans le cadre d'une pratique réflexive.

\section{La pertinence et la richesse du stage en tant que contexte d'enseignement et d'apprentissage pour le développement des compétences professionnelles}

Outre les dimensions biocliniques et techniques spécifiques qu'elle comporte, la pratique de la médecine en contexte de réanimation ou de soins intensifs soulève des enjeux éthiques majeurs 5 . Les situations d'apprentissage rencontrées en stage peuvent ainsi être exploitées pour proposer aux étudiants des objectifs d'apprentissage, formulés en lien avec des compétences en construction, visées à l'issue du stage, en référence avec les principes de l'apprentissage contextualisé́. Le stage permet ainsi de réunir les conditions favorables pour aborder de manière contextualisée des thèmes essentiels tels que : l'obstination déraisonnable, la limitation ou l'arrêt de thérapeutiques actives, la collégialité et la pluridisciplinarité, les dispositions législatives et réglementaires, les principes éthiques et règles déontologiques devant guider l'action du médecin, le refus de traitement et le respect de la volonté du malade. Au-delà de ces aspects, un tel contexte a une portée plus générale, en permettant d'illustrer de façon privilégiée les fondements de la décision médicale en situation d'incertitude et de complexité $e^{7,8}$.

La fréquence des situations où se discutent des décisions de limitation ou d'arrêt de thérapeutiques actives est d'environ une par semaine en moyenne. Ainsi, au cours du stage, chaque étudiant a pu assister à au moins un "staff éthique ", discuter les problèmes posés par ces situations avec les médecins, les internes et les infirmières de l'équipe, au cours des visites quotidiennes et des réunions hebdomadaires de synthèse de la situation de chaque patient et à l'occasion de six gardes en réanimation ; chacun a participé à deux séances d'enseignement avec l'un des enseignants cliniciens, consacrées exclusivement aux problèmes éthiques et liés à la relation médecins/familles (incluant l'annonce d'un décès), dans le contexte particulier de la réanimation.

Le récit rapporté par les quatre étudiantes atteste que, malgré certaines limites - concernant par exemple l'approfondissement qui aurait pu être fait de l'approche de soins palliatifs-, elles ont très bien assimilé l'essentiel de la problématique des décisions de limitation ou d'arrêt de thérapeutiques actives : distinction entre limitation et arrêt de traitements, distinction entre limitation de traitement et euthanasie, refus de l'obstination déraisonnable, recueil et respect des choix de la personne, notions de personne de confiance et de directives anticipées, définition des grands principes éthiques, collégialité de la discussion, nécessité de la continuité des soins dits de confort, prise en compte des dispositions réglementaires. On relève en outre que le questionnement permis par l'exposition à des expériences authentiques a favorisé le développement de démarches d'apprentissage autonome de la part des étudiantes qui ont exploité toutes les ressources à leur disposition, par exemple celles disponibles sur internet, qui leur ont permis de rapporter l'exemple particulier du centre d'éthique clinique de l'hôpital Cochin.

\section{Limportance de rendre explicite le compagnonnage au cours des stages}

Le compagnonnage est souvent tenu comme étant l'un des points forts permis par les activités de stage. On sait aussi que plusieurs limites de ce compagnonnage sont liées à son caractère tacite et non explicite et que des apprentissages non viables, voire carrément non souhaitables, peuvent être la conséquence de l'exposition des étudiants à des contextes professionnels où ils sont mis en situation de devoir faire des inférences implicites, sans bénéficier de rétro-action adéquate' ${ }^{9}$. Ceci est particulièrement le cas dans le domaine des aspects éthiques liés aux actions professionnelles, ce dont rend compte le contexte de curriculum caché ${ }^{10}$.

A cet égard, les "staffs éthiques " constituent à nos yeux une opportunité privilégiée de rendre explicite pour les étudiants les déterminants et le processus délibératif qui fondent les décisions qui sont prises en matière de limitation et d'arrêt de thérapeutiques actives ${ }^{11}$. Le témoignage rapporté illustre bien qu’à partir de la présentation détaillée du dossier du malade, de l'analyse de chaque alternative thérapeutique possible sous le double angle technique et éthique, de l'expression de l'opinion des divers participants, médecins et soignants, qui sont autant d'éléments clairement explicités et débattus, ces " staffs éthiques " permettent de soutenir et de rendre accessible la fonction de modèle de rôle, qui est l'une des modalités essentielles d'intervention des enseignants cliniciens en contexte de stage?.

\section{La contribution des expériences de stage au processus de socialisation professionnelle}

Certains éclairages décrivent comment un "apprenti » migre progressivement d'une situation périphérique vers une position de plus en plus centrale dans un milieu professionnel ${ }^{12}$. A cet égard, les interactions interprofessionnelles permises par le contexte de stage constituent un cadre propice au développement du pro- 


\section{Parole d'étudiants}

cessus de socialisation professionnelle du futur médecin et à son acquisition d'une "culture de pratique". Le récit des étudiantes illustre comment les "staffs éthiques " permettent un partage explicite du questionnement éthique par les différents membres de l'équipe, quels que soient leur grade ou leur fonction ${ }^{4,5,11}$ et en quoi la participation d'étudiants en médecine, mais aussi d'une élève infirmière et parfois d'une élève aidesoignante, est de nature à favoriser la construction de leurs identités professionnelles respectives. D’une manière générale, il souligne l'intérêt de l'intégration des étudiants hospitaliers dans la vie des services pendant les stages mais met aussi en lumière les limites du dispositif français fondé sur un externat à mi-temps exclusif le matin, qui ne leur permet d'avoir qu'une vision parcellaire de la prise en charge du patient. Cette dissociation est préjudiciable à la construction d'une compétence clinique.

\section{Lintérêt de l'écriture pour soutenir des apprentissages dans le cadre d'une pratique réflexive.}

Il est établi que l'écriture est bien plus qu'une activité mécanique et que, exploitée dans le contexte d'un dispositif de formation, elle peut promouvoir l'activité réflexive ${ }^{13}$. Elle favorise notamment une perception plus " objective " des expériences vécues, grâce à un processus de distanciation, en aidant l'étudiant à distinguer les faits et l'interprétation qu'il en a faite ; elle aide l'étudiant à appréhender la composante subjective et affective liée au processus d'apprentissage. Dans l'expérience rapportée, on peut faire l'hypothèse, à titre d'exemple, que l'écriture de leur récit a aidé les étudiantes à analyser avec beaucoup d'authenticité et de profondeur réflexive leurs propres réactions et leur malaise devant un patient sédaté et entouré de technique et qu'elle a facilité leur questionnement et la formulation de leur ressenti face à la souffrance et à la mort et, de façon plus générale, de leur souci des questions touchant au respect de la personne.

\section{Conclusion}

Dans le contexte d'un stage d'externat en réanimation médicale, la confrontation répétée à la mort et à la prise de décisions de limitations ou d'arrêt de thérapeutiques actives a fortement impressionné quatre étudiantes qui étaient invités à en faire un récit dans le cadre d'un travail académique. Leur texte, à la fois narratif et réflexif, illustre l'intérêt des étudiants pour la réflexion éthique concernant les pratiques médicales, dès lors que le contexte de stage est explicitement exploité pour orienter et soutenir des apprentissages intégrant de multiples champs de savoirs. Il met en lumière l'intérêt de faire participer les étudiants à des activités d'élaboration collégiale de décision d'arrêt ou de limitation de traitement, tels que les "staffs éthiques " le permettent, pour rendre explicite certains déterminants et processus de la décision médicale en situation de complexité, d'incertitude et de tension éthique. Il souligne l'intérêt de l'écriture pour soutenir l'apprentissage de la pratique réflexive. Il montre également l'attente des étudiants concernant le partage du questionnement éthique avec les médecins et les personnels soignants des services, en révélant la richesse de la confrontation interprofessionnelle permise dans le cadre des stages. A nos yeux, de telles pistes devraient être davantage exploitées pour améliorer la formation des médecins dans le champ des compétences visant à optimiser la relation médecinmalade ainsi que la prise en compte des préoccupations éthiques en santé, pour lequel plusieurs limites ont été identifiées ${ }^{14}$.

\section{Remerciements}

Nous remercions Jean Jouquan pour ses conseils et sa révision amicale du manuscrit.

\section{Contributions}

Dans le cadre d'un travail acédémique de fin de stage, Marie Sonnier, Bodo Rakotosehno, Catherine Mahé et Corry Droguet ont rédigé conjointement et solidairement le récit rapporté dans cet article. JeanMichel Boles a rédigé la version initiale et finale de l'introduction et de la section "commentaires". Anne Renault a participé aux relectures des versions successives du manuscrit. 


\section{Références}

1. Arrêté du 10 octobre 2000 modifiant l'arrêté du 4 mars 1997 relatif à la deuxième partie du deuxième cycle des études médicales. [On-line]. Disponible sur: http://www.legifrance.gouv.fr

2. Chamberland M. Les séances d'apprentissage du raisonnement clinique $(A R C)$ : un exemple d'activité pédagogique contextualisé adaptée aux stages cliniquesen médecines. An Med Interne 1998;149:479-84.

3. Les limitations et arrêts des thérapeutique(s) active(s) en réanimation adulte : recommandations de la Société de Réanimation de Langue Française. Réanimation 2002;11:442-9.[On-line] Disponible sur : http://www.srlf.org

4. Boles JM. Questions et réflexions sur l'application de la loi du 22 avril 2005. Laennec 2007;3:8-22.

5. Boles JM. Enjeux éthiques en réanimation. In : Ethique, médecine et société (E Hirsch, dir.). Paris : Vuibert (Coll. Espace éthique) 2007:754-61

6. Brown JS, Collins A, Duguid P. Situated cognition and the culture of learning. Educational Researcher 1989; 18:32-42.

7. Mulrow CD, Cook DJ, Davidoff F. Systematic reviews: critical links in the great chain of evidence. Ann Intern Med 1997:126: 389-91.
8. Le Coz P. Petit traité de la décision médicale. Seuil, Paris, 2007.

9. Chamberland M, Hivon R. Les compétences de l'enseignant clinicien et le modèle de rôle en formation clinique. Pédagogie Médicale 2005;6:98-111

10. Hafferty $F W$ Franks $R$. The hidden curriculum, ethics teaching and the structure of medical education. Acad Med 1994;69:861-71.

11. Boles JM. "La place du débat éthique dans la décision médicale en réanimation médicale ». In : Éthique du soin: quels fondements pour quelles pratiques? 1er colloque de l'Espace éthique de Bretagne Occidentale, Presses Universitaires de Rennes, Rennes, 2008 (sous presse).

12. Lave J, Wenger E. Situated Learning: Legitimate peripheral participation. Cambridge (UK): Cambridge University Press, 1991

13. Walker D. Writing in reflection. In: Boud D, Keogh $R$, Walker $D$ (Eds.). Reflection: turning experience into learning. London: Logan, 1985:52-68.

14. Boles JM. Les directives anticipées. Commentaires à propos de la lettre de Jean-Jacques Guilbert. Pédagogie Médicale 2007;8:117-20.

Manuscrit reçu le 15 décembre 2007 ; commentaires éditoriaux formulés aux auteurs le 16 et le 23 janvier 2008 ; accepté pour publication le 24 janvier 2008 